\title{
THE PERTURBATION OF LOVE WAVE SPECTRA
}

\author{
Bx David G. Harkrider
}

\section{ABSTRACT}

The equations governing the variational principles for Love wave spectra are investigated. It is shown that assumptions used by earlier authors are not necessary to the validity of the variational techniques.

Moreover it is demonstrated that except for a homogeneous plate, these assumptions are false for plane multilayered media and lead to incorrect expressions for group-velocity perturbations. The correct expressions are determined and examples of their use are given.

\section{INTRODUCTION}

A potentially important method for the calculation of surface wave dispersion curves and the inversion of observed dispersion data is the use of partial derivatives obtained by techniques based on variational principles. The error in phase velocities calculated in this manner is frequently less than 10 per cent of the difference between the trial and actual values.

On the other hand, group-velocity perturbations using similar techniques yielded velocities which were often closer to the starting or trial values. Motivated by what was felt to be unaccountably large errors for the group velocity, theoretical derivatives were obtained from the dispersion equations of simple models.

While calculating analytic expressions for Love wave energy integrals and their derivatives in a layer over a half-space, it was discovered that unnecessary relations had been used by previous investigators to derive equations for phase-velocity perturbations due to changes in elastic constants. By numerical differentiation, it was verified that Love and Rayleigh wave energy integrals neglected as second order functions of frequency, wave number, or elastic constants in the derivation of group velocity, media response and phase velocity derivatives were actually first order functions of those parameters in a multilayered half-space or plate. The resulting expressions are, fortunately, still correct since the requirement of second order dependence was only a sufficient condition. Unfortunately, the use of these relations give erroneous expressions for the partial derivatives of group velocity and media response for all Love wave models except a homogeneous plate.

In this paper, the relations which govern the variational principles, such as Rayleigh's principle are derived in detail. The previously mentioned relations or assumptions are shown to be unnecessary. Expressions for the plate and the layer over a half-space are derived and values are calculated to show the effect of using the incorrect formulas for group velocity perturbations.

The correct expressions for the perturbations of group velocity and amplitude response are then extended to a multilayered half-space. 


\section{THEORY}

Previous work on the perturbation of Love wave spectra has been based upon variational properties of the Lagrangian

$$
\mathscr{L} \equiv \omega^{2} I_{0}-k^{2} I_{1}-I_{2}
$$

where

$$
\begin{gathered}
I_{0}=\int_{0}^{\infty} \rho v^{2} d z \\
I_{1}=\int_{0}^{\infty} \mu v^{2} d z \\
I_{2}=\int_{0}^{\infty} \mu\left(\frac{d v}{d z}\right)^{2} d z
\end{gathered}
$$

and where $v$ is the horizontal transverse displacement, $\mu$ the rigidity, $\rho$ the density, $k$ the wave number and $\omega$ the frequency. [Meisner, 1926; Jeffreys, 1934, 1961; Takeuchi et al, 1962, 1964; Anderson, 1964; Andrianova et al, 1965; and Vilkovitch et al, 1966]. Before discussing these properties, we will evaluate $\&$ and obtain the associated relations which govern the perturbation equations.

The equation of motion for horizontally polarized shear waves in a media in which the physical parameters $\rho$ and $\mu$ are functions of $z$ only is given by

$$
\frac{d}{d z}\left(\mu \frac{d v}{d z}\right)-k^{2} \mu v+\omega^{2} \rho v=0
$$

Multiplying equation (5) by $v$ and integrating from $z=0$ tò $\infty$ yields the desired form

$$
\mathcal{L}=-\left.\left(v \mu \frac{d v}{d z}\right)\right|_{0} ^{\infty}
$$

In obtaining equation (6), the integration of

$$
\int_{0}^{\infty} v \frac{d}{d z}\left(\mu \frac{d v}{d z}\right) d z
$$

was done by parts and by making use of the continuity of displacement, $v$, and stress, $\mu d v / d z$, at discontinuities of the elastic parameters.

For Love waves, we impose the boundary conditions of a free surface at $z=0$, i.e., $\mu(0) d v^{(0)} / d z=0$ and that the motion vanish at infinity. Therefore for Love waves in a vertically heterogeneous half-space, the Lagrangian vanishes.

$$
\mathfrak{L}(\omega, k, \mu, \rho)=0 \text {. }
$$


For a heterogeneous plate, bounded at say $z=0$ and $z=H$ by either a free or rigid surface, i.e., $\mu d v / d z=0$ or $v=0$ respectively, we obtain the same result.

$$
\omega^{2} I_{0}=k^{2} I_{1}+I_{2} .
$$

Similarly, if we multiply equation $(5)$ by $(\partial v / \partial p)$ and integrate from $z=0$ to $z=\infty$ where $p$ is one of the variables $\omega, k, \mu$ or $\rho$ while keeping the other three constant, we obtain

$$
\begin{aligned}
\omega^{2} \int_{0}^{\infty} \rho \frac{\partial}{\partial p}\left(v^{2}\right) d z=k^{2} \int_{0}^{\infty} \mu & \frac{\partial}{\partial p}\left(v^{2}\right) d z \\
& \quad+\int_{0}^{\infty} \mu \frac{\partial}{\partial p}\left[\left(\frac{d v}{d z}\right)^{2}\right] d z-\left.2\left(\frac{\partial v}{\partial p} \mu \frac{d v}{d z}\right)\right|_{0} ^{\infty}
\end{aligned}
$$

where we have used the continuity of $\partial v / \partial p$ across interfaces. Any algorithm which makes use of the continuity of displacement in numerical calculations of the eigenfunctions will ensure this. Again the boundary conditions at $z=0$ and infinity require that the last term in equation (9) vanish. Thus equation (9) becomes

$$
\omega^{2} \int_{0}^{\infty} \rho \frac{\partial}{\partial p}\left(v^{2}\right) d z=k^{2} \int_{0}^{\infty} \mu \frac{\partial}{\partial p}\left(v^{2}\right) d z+\int_{0}^{\infty} \mu \frac{\partial}{\partial p}\left[\left(\frac{d v}{d z}\right)^{2}\right] d z
$$

As we shall see, these four equations (10) govern the perturbation equations for Love wave spectra in vertically heterogeneous media. First let us consider the perturbation due to a small change in wave number, sk. The resulting perturbation of the integrands can be expressed as

$$
\delta \int A d z=\int \delta A d z=\left[\int\left(\frac{\partial A}{\partial k}\right)_{\omega} d z+U \int\left(\frac{\partial A}{\partial \omega}\right)_{k} d z\right] \delta k+O(\delta k)^{2}
$$

where the group velocity $U$ is

$$
U=\frac{d \omega}{d k}
$$

Combining this with equations $(10)$ for $p=\omega$ and $k$, yields

$$
\omega^{2} \int \rho \delta\left(v^{2}\right) d z=k^{2} \int \mu \delta\left(v^{2}\right) d z+\int \mu \delta\left[\left(\frac{d v}{d z}\right)^{2}\right] d z+O(\delta k)^{2}
$$

or

$$
\omega^{2} \delta I_{0}=k^{2} \delta I_{1}+\delta I_{2}+O(\delta k)^{2} .
$$

The energy equation (8) for the new eigensolutions is

$$
(\omega+\delta \omega)^{2}\left(I_{0}+\delta I_{0}\right)=(k+\delta k)^{2}\left(I_{1}+\delta I_{1}\right)+I_{2}+\delta I_{2} .
$$


Expanding gives

$$
(\omega+\delta \omega)^{2} I_{0}+\omega^{2} \delta I_{0}=(k+\delta k)^{2} I_{1}+k^{2} \delta I_{1}+I_{2}+\delta I_{2}+O(\delta k)^{2}
$$

and using equation (12) we obtain the expression of Rayleigh's principle found in Jeffreys (1961)

$$
(\omega+\delta \omega)^{2} I_{0}=(k+\delta k)^{2} I_{1}+I_{2}+O(\delta k)^{2}
$$

which in turn yields

$$
U=\frac{d \omega}{d k}=\frac{I_{1}}{c I_{0}}
$$

For the perturbation due to a small change in rigidity $\delta \mu$, keeping $\omega$ and $\rho$ fixed, we can write the integrand perturbations as

$$
\int \delta A_{\mu} d z=\left[\int\left(\frac{\partial A}{\partial \mu}\right)_{\omega, k} d z+\left(\frac{\partial k}{\partial \mu}\right)_{\omega} \int\left(\frac{\partial A}{\partial k}\right)_{\mu} d z\right] \delta \mu+O(\delta \mu)^{2}
$$

Using equation (15) with equation (10) where $p=\mu$, we obtain

$$
\omega^{2} \int_{:} \rho \delta\left(v^{2}\right)_{\mu} d z=k^{2} \int \mu \delta\left(v^{2}\right)_{\mu} d z+\int \mu \delta\left[\left(\frac{d v}{d z}\right)^{2}\right]_{\mu} d z+O(\delta \mu)^{2} .
$$

The energy equation (8) becomes

$$
\omega^{2}\left[I_{0}+\delta\left(I_{0}\right)_{\mu}\right]=\left(k+\delta k_{\mu}\right)^{2}\left[I_{1}+\delta\left(I_{1}\right)_{\mu}\right]+I_{2}+\delta\left(I_{2}\right)_{\mu}
$$

Neglecting terms higher than first order in $\delta \mu$ and $\delta k$ equation (17) reduces to

$$
\omega^{2} I_{0}+\omega^{2} \delta\left(I_{0}\right)_{\mu}=k^{2} I_{1}+k^{2} \delta\left(I_{1}\right)_{\mu}+2 k \delta k_{\mu} I_{1}+I_{2}+\delta\left(I_{2}\right)_{\mu}
$$

and by equations (8) and (16) to

$$
0=k^{2} \int \delta \mu \cdot v^{2} d z+2 k \delta k_{\mu} I_{1}+\int \delta \mu \cdot\left(\frac{d v}{d z}\right)^{2} d z
$$

or

$$
\delta c_{\mu}=-\frac{c}{k} \delta k_{\mu}=\frac{c}{2 k^{2} I_{1}}\left[k^{2} \int \delta \mu \cdot v^{2} d z+\int \delta \mu \cdot\left(\frac{d v}{d z}\right)^{2} d z\right]
$$

where we have used the relations

$$
\delta\left(I_{0}\right)_{\mu}=\int \rho \delta\left(v^{2}\right)_{\mu} d z
$$




$$
\begin{aligned}
& \delta\left(I_{1}\right)_{\mu}=\int \delta \mu \cdot v^{2} d z+\int \mu \cdot \delta\left(v^{2}\right)_{\mu} d z \\
& \delta\left(I_{2}\right)_{\mu}=\int \delta \mu\left(\frac{d v}{d z}\right)^{2} d z+\int \mu \cdot \delta\left[\left(\frac{d v}{d z}\right)^{2}\right]_{\mu} d z .
\end{aligned}
$$

Similarly for a small perturbation in density $\delta \rho$ with $\omega$ and $\mu$ fixed we obtain

$$
\delta c_{\rho}=-\frac{c^{2}}{2 I_{1}} \int \delta \rho \cdot v^{2} d z
$$

These are the same expressions obtained in Anderson (1964), Andrianova et al (1965) and Vilkovitch et al (1966) by assuming that the relations

$$
\int \rho \delta\left(v^{2}\right)_{p} d z, \quad \int \mu \delta\left(v^{2}\right)_{p} d z \text { and } \int \mu \delta\left[\left(\frac{d v}{d z}\right)^{2}\right]_{o} d z
$$

are second order in $\delta p$ where $p$ can be $k, \omega, \rho$ or $\mu$, i.e.,

$$
\int \rho \frac{\partial}{\partial p}\left(v^{2}\right) d z=\int \mu \frac{\partial}{\partial p}\left(v^{2}\right) d z=\int \mu \frac{\partial}{\partial p}\left[\left(\frac{d v}{d z}\right)^{2}\right] d z=0
$$

By using equations (10) to obtain the same result, we see that these assumptions are not necessary for the Rayleigh principle to be valid. In fact, we will demonstrate in the next section that except for a single layer plate these conditions are false for plane multilayered media. Although of no consequence in deriving formulas for $U, \delta c \mu$ and $\delta c \rho$ these invalid assumptions yield incorrect expressions for the group-velocity perturbations in Vilkovitch et al (1965). As of yet the spherical case has not been investigated but it would be very fortuitous if the group velocity partial derivatives for a sphere in Vilkovitch et al (1966) and Andrianova et al (1965) were correct while the corresponding expressions for the half-space and heterogeneous plate were not. Backus and Gilbert (1967) state that the integrals of the variations of the spherical eigenfunctions sum out to zero and that the individual integrals are not necessarily zero for similar relations in the spherical problem.

In a multilayered structure of homogeneous layers, the energy integrals take the form

$$
\begin{aligned}
& I_{0}=\sum_{j=1}^{n} \rho_{j} \int_{z_{j-1}}^{z_{j}} v^{2} d z \equiv \sum_{j=1}^{n} \rho_{j} D_{j} \\
& I_{1}=\sum_{j=1}^{n} \mu_{j} \int_{z_{j-1}}^{z_{j}} v^{2} d z \equiv \sum_{j=1}^{n} \mu_{j} D_{j} \\
& I_{2}=\sum_{j=1}^{n} \mu_{j} \int_{z_{j-1}}^{z_{j}}\left(\frac{d v}{d z}\right)^{2} d z \equiv \sum_{j=1}^{n} \mu_{j} S_{j}
\end{aligned}
$$


and equations (10) can be written

$$
\begin{aligned}
& \omega^{2} \sum_{j=1}^{n} \rho_{j}\left(\frac{\partial D_{j}}{\partial k}\right)_{\omega, \mu, \rho}=k^{2} \sum_{j=1}^{n} \mu_{j}\left(\frac{\partial D_{j}}{\partial k}\right)_{\omega, \mu, \rho}+\sum_{j=1}^{n} \mu_{j}\left(\frac{\partial S_{j}}{\partial k}\right)_{\omega, \mu, \rho} \\
& \omega^{2} \sum_{j=1}^{n} \rho_{j}\left(\frac{\partial D_{j}}{\partial \omega}\right)_{k, \mu, \rho}=k^{2} \sum_{j=1}^{n} \mu_{j}\left(\frac{\partial D_{j}}{\partial \omega}\right)_{k, \mu, \rho}+\sum_{j=1}^{n} \mu_{j}\left(\frac{\partial S_{j}}{\partial \omega}\right)_{k, \mu, \rho} \\
& \omega^{2} \sum_{j=1}^{n} \rho_{j}\left(\frac{\partial D_{j}}{\partial \rho_{m}}\right)_{\omega, k, \mu}=k^{2} \sum_{j=1}^{n} \mu_{j}\left(\frac{\partial D_{j}}{\partial \rho_{m}}\right)_{\omega, k, \mu}+\sum_{j=1}^{n} \mu_{j}\left(\frac{\partial S_{j}}{\partial \rho_{m}}\right)_{\omega, k, \mu} \\
& \omega^{2} \sum_{j=1}^{n} \rho_{j}\left(\frac{\partial D_{j}}{\partial \mu_{m}}\right)_{\omega, k, \rho}=k^{2} \sum_{j=1}^{n} \mu_{j}\left(\frac{\partial D_{j}}{\partial \mu_{m}}\right)_{\omega, k, \rho}+\sum_{j=1}^{n} \mu_{j}\left(\frac{\partial S_{j}}{\partial \mu_{m}}\right)_{\omega, k, \rho}
\end{aligned}
$$

where it is understood that in taking the derivatives with respect to a particular layer $\rho_{m}$ and $\mu_{m}$ that the other Iayer $\rho$ ' $\$$ and $\mu$ 's are held constant.

From equations (24) we can obtain the partial derivatives of the Lagrangian

$$
\begin{aligned}
& \left(\frac{\partial \mathscr{L}}{\partial k}\right)_{\omega, \rho, \mu}=-2 k I_{1} \\
& \left(\frac{\partial \mathscr{L}}{\partial \omega}\right)_{k, \rho, \mu}=2 \omega I_{0} \\
& \left(\frac{\partial \mathscr{L}}{\partial \rho_{m}}\right)_{\omega, k, \mu}=\omega^{2} D_{m} \\
& \left(\frac{\partial \mathscr{L}}{\partial \mu_{m}}\right)_{\omega, k, \rho}=-\left(k^{2} D_{m}+S_{m}\right) .
\end{aligned}
$$

Making use of the implicit relation

$$
\mathscr{L}(\omega, k, \mu, \rho)=0
$$

it is easy to show that

$$
\begin{aligned}
U & =-\left(\frac{\partial \mathcal{L}}{\partial k}\right)_{\omega, \rho, \mu} /\left(\frac{\partial \mathcal{L}}{\partial \omega}\right)_{k, \rho, \mu}=\frac{I_{1}}{c I_{0}} \\
\left(\frac{\partial c}{\partial \rho_{m}}\right)_{\omega, \mu} & =-\frac{c^{3} D_{m}}{2 I_{1}}=\frac{c}{U}\left(\frac{\partial c}{\partial \rho_{m}}\right)_{k, \mu} \\
\left(\frac{\partial c}{\partial \mu_{m}}\right)_{\omega, \rho} & =\frac{c}{k^{2}} \frac{\left(k^{2} D_{m}+S_{m}\right)}{2 I_{1}}=\frac{c}{U}\left(\frac{\partial c}{\partial \mu_{m}}\right)_{k, \rho}
\end{aligned}
$$

(Anderson and Harkrider, 1968). These expressions are equivalent to those given in Anderson (1964) and Vilkovitch et al (1966). 
The Lagrangian for each homogeneous layer, $\mathfrak{L}_{j}$, can be evaluated in the same manner as equation (6) to give

$$
\mathfrak{L}_{j}=-\left(v_{j} \tau_{j}-v_{j-1} \tau_{j-1}\right)
$$

where $v_{j}$ is the horizontal displacement and $\tau_{j}$ the tangential stress at the bottom of the $j$ th layer. For the half-space, i.e., layer $n$, the stress is related to the displacement by

$$
\tau_{n}(z)=\mu_{n} k r_{\beta n} v_{n}(z)
$$

where

and the shear velocity

$$
r_{\beta n}^{*}=-\left(1-\frac{c^{2}}{\beta_{n}{ }^{2}}\right)^{1 / 2}
$$

$$
\beta_{n}{ }^{2}=\frac{\mu_{n}}{\rho_{n}}
$$

The Lagrangian of the half-space is then

$$
\mathfrak{L}_{n}=\tau_{n-1} v_{n-1}=\mu_{n} k r_{\beta_{n}}^{*} v_{n-1}^{2} .
$$

In terms of the Thomson-Haskell matrix formulation equations (27) and (29) become

$$
\mathfrak{L}_{j}=k\left[\left(A_{j}\right)_{11}\left(A_{j}^{*}\right)_{21}-\left(A_{j-1}\right)_{11}\left(A_{j-1}^{*}\right)_{21}\right]
$$

and

$$
\mathfrak{L}_{n}=k \mu_{n} r_{\beta n}^{*}\left(A_{n-1}\right)_{11}^{2}
$$

where $\left(A_{j}\right)_{11}$ and $\left(A_{j}{ }^{*}\right)_{21}$ are elements of the Thomson-Haskell multilayer matrix for the first $j$ layers. The Lgrangian of the entire system for Love waves is

$$
\mathscr{L}=\sum_{j=1}^{n} \mathfrak{L}_{j}=k\left(A_{n-1}\right)_{11}\left[\left(A_{n-1}^{*}\right)_{21}+\mu_{n} r_{\beta n}^{*}\left(A_{n-1}\right)_{11}\right]
$$

since $\left(A_{0}{ }^{*}\right)_{21}$ is initialized to zero in the Thomson-Haskell algorithm.

From Harkrider (1964) the spectral amplitude response for a surface source and receiver at a distance of one wavelength for Love waves is given by

$$
A=\frac{1}{\left(A_{n-1}\right)_{11}\left(\frac{\partial F_{L}}{\partial k}\right)_{\omega}}
$$

where

$$
F_{L}=-\left[\left(A_{n-1}^{*}\right)_{21}+\mu_{n} r_{\beta n}^{*}\left(A_{n-1}\right)_{11}\right] .
$$


Comparing equations (34) and (32), we see that

$$
\mathcal{L}=-k\left(A_{n-1}\right)_{11} F_{L}
$$

and

$$
\begin{aligned}
\left(\frac{\partial \mathcal{L}}{\partial k}\right)_{\omega} & =-\left\{\left(A_{n-1}\right)_{11}+k\left[\frac{\partial\left(A_{n-1}\right)_{11}}{\partial k}\right]_{\omega}\right\}_{L}-k\left(A_{n-1}\right)_{11}\left(\frac{\partial F_{L}}{\partial k}\right)_{\omega} \\
& =-k\left(A_{n-1}\right)_{11}\left(\frac{\partial F_{L}}{\partial h}\right)_{\omega}
\end{aligned}
$$

where the period equation $F_{L}(\omega, k)=0$ determines the relationship between $\omega$ and $k$ for Love waves. Combining equations (36), (33) and (25) yields an expression for the amplitude response in terms of an energy integral, i.e.,

$$
A=\frac{1}{2 I_{1}}
$$

(Harkrider and Anderson, 1966). This relationship is also valid for the free or rigid multilayered plate. Equation (37) can be obtained in a more elegant and straightforward manner from the inhomogeneous form of the differential equation (5). (Neigauz in Tieilis-Borok and Yanovskaya, 1962; Andrianova et al, 1965; Vlaar, 1966; and Saito, 1967).

Similar relations to equation (10) are also valid for Rayleigh waves, but will not be discussed in this paper.

\section{Analytical and Numerical Results}

For a homogeneous plate of thickness $d_{1}$ the energy integrals are given by

$$
\begin{aligned}
& I_{0}=\rho_{1} \frac{d_{1}}{2}\left[1+\cos Q_{1} \frac{\sin Q_{1}}{Q_{1}}\right] \\
& I_{1}=\mu_{1} \frac{d_{1}}{2}\left[1+\cos Q_{1} \frac{\sin Q_{1}}{Q_{1}}\right] \\
& I_{2}=\mu_{1}\left(\frac{\omega^{2}}{\beta_{1}^{2}}-k^{2}\right) \frac{d_{1}}{2}\left[1-\cos Q_{1} \frac{\sin Q_{1}}{Q_{1}}\right] .
\end{aligned}
$$

If the plate is bounded on both sides by a free surface the period equation is

$$
\sin Q_{1}=0
$$

where

$$
Q_{1}=k r_{\beta 1} d_{1}
$$

and

$$
r_{\beta 1}=\left(\frac{c^{2}}{\beta_{1}{ }^{2}}-1\right)^{1 / 2}
$$


Substitution of equation (39) in equations (38) yields

$$
\begin{aligned}
& I_{0}=\rho_{1} \frac{d_{1}}{2} \\
& I_{1}=\mu_{1} \frac{d_{1}}{2} \\
& I_{2}=\mu_{1}\left(\frac{\omega^{2}}{\beta_{1}^{2}}-k^{2}\right) \frac{d_{1}}{2}
\end{aligned}
$$

and from equations (26) and (37) we obtain

$$
U=\frac{\beta_{1}^{2}}{c}
$$

and

$$
A=\frac{1}{\mu_{1} d_{1}} .
$$

It is obvious that

$$
\frac{d I_{0}}{d k}=\frac{d I_{0}}{d \omega}=\frac{d I_{0}}{d \mu_{1}}=0
$$

and

$$
\frac{d I_{1}}{d k}=\frac{d I_{1}}{d \omega}=\frac{d I_{1}}{d \rho_{1}}=0
$$

which agree with the assumptions of Anderson (1964), Andrianova et al (1965), and Vilkovitch et al (1966).

For the case of a layer over a half-space the energy integrals are

$$
\begin{aligned}
& I_{0}=\rho_{1} \frac{d_{1}}{2}\left\{1+\cos Q_{1} \frac{\sin Q_{1}}{Q_{1}}\right\}-\frac{\rho_{2}}{2} \frac{\cos ^{2} Q_{1}}{k r_{\beta 2}^{*}} \\
& I_{1}=\mu_{1} \frac{d_{1}}{2}\left\{1+\cos Q_{1} \frac{\sin Q_{1}}{Q_{1}}\right\}-\frac{\mu_{2}}{2} \frac{\cos ^{2} Q_{1}}{k r_{\beta 2}^{*}} \\
& I_{2}=\mu_{1} \frac{Q_{1}^{2}}{2 d_{1}}\left\{1-\cos Q_{1} \frac{\sin Q_{1}}{Q_{1}}\right\}-\frac{\mu_{2}}{2} k r_{\beta 2}^{*} \cos ^{2} Q_{1}
\end{aligned}
$$

The classical Love wave period equation for this case is

$$
\tan Q_{1}=-\frac{\mu_{2} r_{\beta 2}^{*}}{\mu_{1} r_{\beta 1}}
$$


Since $I_{2}$ is not involved in calculating perturbations of $U$ and $A$, we will restrict the following to integrals $I_{0}$ and $I_{1}$. Evaluating $I_{0}$ and $I_{1}$ for values of $\omega$ and $k$ given by

TABLE 1

Layer Thickness (D), Shear Velocity (Beta), Denstty (RHo) and Rigrdity (MU) For the STandard aND Perturbed One Layer Models

\begin{tabular}{ccccc}
\hline $\mathrm{D}(\mathrm{km})$ & \multicolumn{1}{c}{ Beta $(\mathrm{km} / \mathrm{sec})$} & $\mathrm{Rho}\left(\mathrm{g} / \mathrm{cm}^{3}\right)$ & $\mathrm{Mu}\left(10^{10} \mathrm{dy} / \mathrm{cm}^{2}\right)$ \\
\hline & \multicolumn{4}{c}{ Standard } \\
40. & 3.6 & 2.8 & 36.288 \\
& 4.5 & 3.3 & 66.825 \\
& & Perturbed & \\
40. & 3.8 & 3.0 & 43.320 \\
& 4.5 & 3.3 & 66.825 \\
\hline
\end{tabular}

TABLE 2

Phase Velocities (c), Group Veloctmies (U), and Amplitude Responses (A) For the Standard One Layer Model

$\begin{array}{rccc}\mathrm{T}(\mathrm{sec}) & \mathrm{c}(\mathrm{km} / \mathrm{sec}) & \mathrm{U}(\mathrm{km} / \mathrm{sec}) & \mathrm{A}\left(10^{-15} \mu / \mathrm{dy}\right) \\ 120.13 & 4.4550 & 4.3677 & 0.2591 \\ 113.90 & 4.4500 & 4.3534 & 0.2896 \\ 103.83 & 4.4400 & 4.3250 & 0.3514 \\ 95.99 & 4.4300 & 4.2970 & 0.4145 \\ 89.65 & 4.4200 & 4.2694 & 0.4788 \\ 79.90 & 4.4000 & 4.2154 & 0.6108 \\ 69.64 & 4.3700 & 4.1377 & 0.8167 \\ 64.51 & 4.3500 & 4.0883 & 0.9587 \\ 55.06 & 4.3000 & 3.9733 & 1.3276 \\ 48.35 & 4.2500 & 3.8713 & 1.7119 \\ 43.16 & 4.2000 & 3.7825 & 2.1059 \\ 38.90 & 4.1500 & 3.7068 & 2.5043 \\ 35.24 & 4.1000 & 3.6440 & 2.9024 \\ 28.99 & 4.0000 & 3.5544 & 3.6820 \\ 23.45 & 3.9000 & 3.5089 & 4.4228 \\ 18.01 & 3.8000 & 3.5027 & 5.1204 \\ 11.96 & 3.7000 & 3.5324 & 5.8013 \\ 6.70 & 3.6350 & 3.5711 & 6.3047 \\ 3.45 & 3.6100 & 3.5909 & 6.5887\end{array}$

equation (45) yields

$$
\begin{aligned}
& I_{0}=\frac{d_{1}}{2}\left[\rho_{1}-\frac{\cos ^{2} Q_{1}}{Q_{1}}\left(\rho_{1} \frac{\mu_{2} r_{\beta 2}^{*}}{\mu_{1} r_{\beta 1}}+\rho_{2} \frac{r_{\beta 1}}{r_{\beta 2}^{*}}\right)\right] \\
& I_{1}=\frac{d_{1}}{2}\left[\mu_{1}-\frac{\cos ^{2} Q_{1}}{Q_{1}} \mu_{2}\left(\frac{r_{\beta 2}^{*}}{r_{\beta 1}}+\frac{r_{\beta 1}}{r_{\beta 2}^{*}}\right)\right] .
\end{aligned}
$$


In order to calculate the derivatives of equations (46), the following formulas were used

$$
\begin{gathered}
\frac{d I_{l}}{d k}=\left(\frac{\partial I_{l}}{\partial k}\right)_{\omega}+U\left(\frac{\partial I_{l}}{\partial \omega}\right)_{k} \\
\left(\frac{\partial I_{l}}{\partial \mu_{1}}\right)_{\omega}=\left(\frac{\partial I_{l}}{\partial \mu_{1}}\right)_{\omega, k}+\left(\frac{\partial k}{\partial \mu_{1}}\right)_{\omega}\left(\frac{\partial I_{l}}{\partial k}\right)_{\omega, \mu}
\end{gathered}
$$

and

$$
\left(\frac{\partial I_{l}}{\partial \rho_{1}}\right)_{\omega}=\left(\frac{\partial I_{l}}{\partial \rho_{1}}\right)_{\omega, k}+\left(\frac{\partial k}{\partial \rho_{1}}\right)_{\omega}\left(\frac{\partial I_{l}}{\partial k}\right)_{\omega, \rho}
$$

where by equations (26)

$$
\begin{aligned}
U & =\frac{\left[\mu_{1}-\frac{\cos ^{2} Q_{1}}{Q_{1}} \mu_{2}\left(\frac{r_{\beta 2}^{*}}{r_{\beta 1}}+\frac{r_{\beta 1}}{r_{\beta 2}^{*}}\right)\right]}{c\left[\rho_{1}-\frac{\cos ^{2} Q_{1}}{Q_{1}}\left(\rho_{1} \frac{\mu_{2}}{\mu_{1}} \frac{r_{\beta 2}^{*}}{r_{\beta 1}}+\rho_{2} \frac{r_{\beta 1}}{r_{\beta 2}^{*}}\right)\right]} \\
\left(\frac{\partial k}{\partial \mu_{1}}\right)_{\omega} & =-\frac{k}{c}\left(\frac{\partial c}{\partial \mu_{1}}\right)_{\omega} \\
& =-\frac{k\left[\left(1-\frac{\cos ^{2} Q_{1}}{Q_{1}} \frac{\mu_{2}}{\mu_{1}} \frac{r_{\beta 2}^{*}}{r_{\beta 1}}\right)+r_{\beta 1}^{2}\left(1+\frac{\cos ^{2} Q_{1}}{Q_{1}} \frac{\mu_{1}}{\mu_{2}} \frac{r_{\beta 2}^{*}}{r_{\beta 1}}\right)\right]}{\left.\mu_{1}-\frac{\cos ^{2} Q_{1}}{Q_{1}} \mu_{2}\left(\frac{r_{\beta 2}^{*}}{r_{\beta 1}}+\frac{r_{\beta 1}}{r_{\beta 2}^{*}}\right)\right]} \\
\left(\frac{\partial k}{\partial \rho_{1}}\right)_{\omega}^{*} & =-\frac{k}{c}\left(\frac{\partial c}{\partial \rho_{1}}\right)_{\omega} \\
& =\frac{\omega^{2}\left(1-\frac{\cos ^{2} Q_{1}}{Q_{1}} \frac{\mu_{2}}{\mu_{1}} \frac{r_{\beta 2}^{*}}{r_{\beta 1}}\right)}{2 k\left[\mu_{1}-\frac{\cos ^{2} Q_{1}}{Q_{1}} \mu_{2}\left(\frac{r_{\beta 2}^{*}}{r_{\beta 1}}+\frac{r_{\beta 1}}{r_{\beta 2}^{*}}\right)\right]}
\end{aligned}
$$

and

$$
l=0 \quad \text { or } \quad 1 \text {. }
$$

Using these equations, partial derivatives of the integrals $I_{0}$ and $I_{1}$ were calculated for a standard layer over half-space model. For comparison purposes, this model is the same as used in Vilkovitch et al (1966). The physical parameters are listed in Table 1, and the spectral values for various periods are given in Table 2. 
The numerical results showed that

$$
\begin{aligned}
\frac{d I_{0}}{d k} \neq 0, & \frac{d I_{1}}{d k} \neq 0, \quad \frac{d I_{2}}{d k} \neq 0 \\
\frac{d I_{0}}{d \omega} \neq 0, & \frac{d I_{1}}{d \omega} \neq 0, \quad \frac{d I_{2}}{d \omega} \neq 0 \\
\left(\frac{\partial I_{0}}{\partial \mu_{1}}\right)_{\omega} \neq 0, & \left(\frac{\partial I_{0}}{\partial \rho_{1}}\right)_{\omega} \neq D_{1}, \quad\left(\frac{\partial I_{1}}{\partial \mu_{1}}\right)_{\omega} \neq D_{1} \\
\left(\frac{\partial I_{1}}{\partial \rho_{1}}\right)_{\omega} \neq 0, & \left(\frac{\partial I_{2}}{\partial \rho_{1}}\right)_{\omega} \neq 0 \text { and } \quad\left(\frac{\partial I_{2}}{\partial \mu_{1}}\right)_{\omega} \neq S_{1}
\end{aligned}
$$

which are in direct conflict with the assumptions of Anderson (1964) and Vilkovitch et al (1966).

These results require no changes in the formulas for phase-velocity perturbations given in Anderson (1964) and Vilkovitch et al (1966). However, additional terms must be added to the expressions for group-velocity perturbations in Vilkovitch et al (1966).

For a multilayered media, the correct expressions are

$$
\left(\frac{\partial c}{\partial \mu_{m}}\right)_{\omega, \rho}=\frac{c}{k^{2}} \frac{\left(k^{2} D_{m}+S_{m}\right)}{2 I_{1}}
$$

$$
\left(\frac{\partial c}{\partial \rho_{m}}\right)_{\omega, \mu}=-\frac{c^{3} D_{m}}{2 I_{1}}
$$$$
\left(\frac{\partial c}{\partial \beta_{m}}\right)_{\omega, \rho}=\frac{c}{2 I_{1}}\left[2 \beta_{m} \rho_{m} D_{m}+2 \frac{\beta_{m} \rho_{m}}{k^{2}} S_{m}\right]
$$$$
\left(\frac{\partial c}{\partial \rho_{m}}\right)_{\omega, \mu}=\frac{c}{2 I_{1}}\left[\beta_{m}^{2} D_{m}+\frac{\beta_{m}{ }^{2}}{k^{2}} S_{m}-c^{2} D_{m}\right]
$$

$$
\begin{aligned}
&\left(\frac{\partial U}{\partial \mu_{m}}\right)_{\omega, \rho}=\frac{1}{c I_{0}}\left[D_{m}+\sum_{j=1}^{n} \mu_{j}\left(\frac{\partial D_{j}}{\partial \mu_{m}}\right)_{\omega, \rho}\right]-\frac{U}{I_{0}} \sum_{j=1}^{n} \rho_{j}\left(\frac{\partial D_{j}}{\partial \mu_{m}}\right)_{\omega, \rho}-\frac{U}{c}\left(\frac{\partial c}{\partial \mu_{m}}\right)_{\omega, \rho} \\
&\left(\frac{\partial U}{\partial \rho_{m}}\right)_{\omega, \mu}= \frac{1}{c I_{0}} \sum_{j=1}^{n} \mu_{j}\left(\frac{\partial D_{j}}{\partial \rho_{m}}\right)_{\omega, \mu}-\frac{U}{I_{0}}\left[D_{m}+\sum_{j=1}^{n} \rho_{j}\left(\frac{\partial D_{j}}{\partial \rho_{m}}\right)_{\omega, \mu}\right]-\frac{U}{c}\left(\frac{\partial c}{\partial \rho_{m}}\right)_{\omega, \mu} \\
&\left(\frac{\partial U}{\partial \beta_{m}}\right)= \frac{2 \beta_{m} \rho_{m}}{c I_{0}}\left[D_{m}+\sum_{j=1}^{n} \mu_{j}\left(\frac{\partial D_{j}}{\partial \mu_{m}}\right)_{\omega, \rho}\right] \\
&-\frac{2 \beta_{m} \rho_{m} U}{I_{0}} \sum_{j=1}^{n} \rho_{j}\left(\frac{\partial D_{j}}{\partial \mu_{m}}\right)_{\omega, \rho}-\frac{U}{c}\left(\frac{\partial c}{\partial \beta_{m}}\right)_{\omega, \rho}
\end{aligned}
$$




$$
\begin{aligned}
\left(\frac{\partial U}{\partial \rho_{m}}\right)_{\omega, \beta}= & \frac{1}{c I_{0}}\left\{\beta_{m}{ }^{2} D_{m}+\sum_{j=1}^{n} \mu_{j}\left[\left(\frac{\partial D_{j}}{\partial \rho_{m}}\right)_{\omega, \mu}+\beta_{m}{ }^{2}\left(\frac{\partial D_{j}}{\partial \mu_{m}}\right)_{\omega, \rho}\right]\right\} \\
& -\frac{U}{I_{0}}\left\{D_{m}+\sum_{j=1}^{n} \rho_{j}\left[\left(\frac{\partial D_{j}}{\partial \rho_{m}}\right)_{\omega, \mu}+\beta_{m}{ }^{2}\left(\frac{\partial D_{j}}{\partial \mu_{m}}\right)_{\omega, \rho}\right]\right\}-\frac{U}{c}\left(\frac{\partial c}{\partial \rho_{m}}\right)_{\omega, \beta}
\end{aligned}
$$

and

$$
\begin{aligned}
& \left(\frac{\partial A}{\partial \mu_{m}}\right)_{\omega, \rho}=-\frac{A}{I_{0}}\left[D_{m}+\sum_{j=1}^{n} \mu_{j}\left(\frac{\partial D_{j}}{\partial \mu_{m}}\right)_{\omega, \rho}\right] \\
& \left(\frac{\partial A}{\partial \rho_{m}}\right)_{\omega, \mu}=-\frac{A}{I_{1}} \sum_{j=1}^{n} \mu_{j}\left(\frac{\partial D_{j}}{\partial \rho_{m}}\right)_{\omega, \mu} \\
& \left(\frac{\partial A}{\partial \beta_{m}}\right)_{\omega, \rho}=-\frac{2 \beta_{m} \rho_{m} A}{I_{1}}\left[D_{m}+\sum_{j=1}^{n} \mu_{j}\left(\frac{\partial D_{j}}{\partial \mu_{m}}\right)_{\omega, \rho}\right] \\
& \left(\frac{\partial A}{\partial \rho_{m}}\right)_{\omega, \beta}=-\frac{A}{I_{1}}\left\{\beta_{m}{ }^{2}\left[D_{m}+\sum_{j=1}^{n} \mu_{j}\left(\frac{\partial D_{j}}{\partial \mu_{m}}\right)_{\omega, \rho}\right]+\sum_{j=1}^{n} \mu_{j}\left(\frac{\partial D_{j}}{\partial \rho_{m}}\right)_{\omega, \mu}\right\} .
\end{aligned}
$$

Setting the derivatives of $D_{j}$ to zero in equation (50), yields the Vilkovitch et al (1966) formulas for group-velocity perturbations.

As a check on the integral formulation program, the group velocities and the perturbations of phase and group velocity were calculated by the following alternate

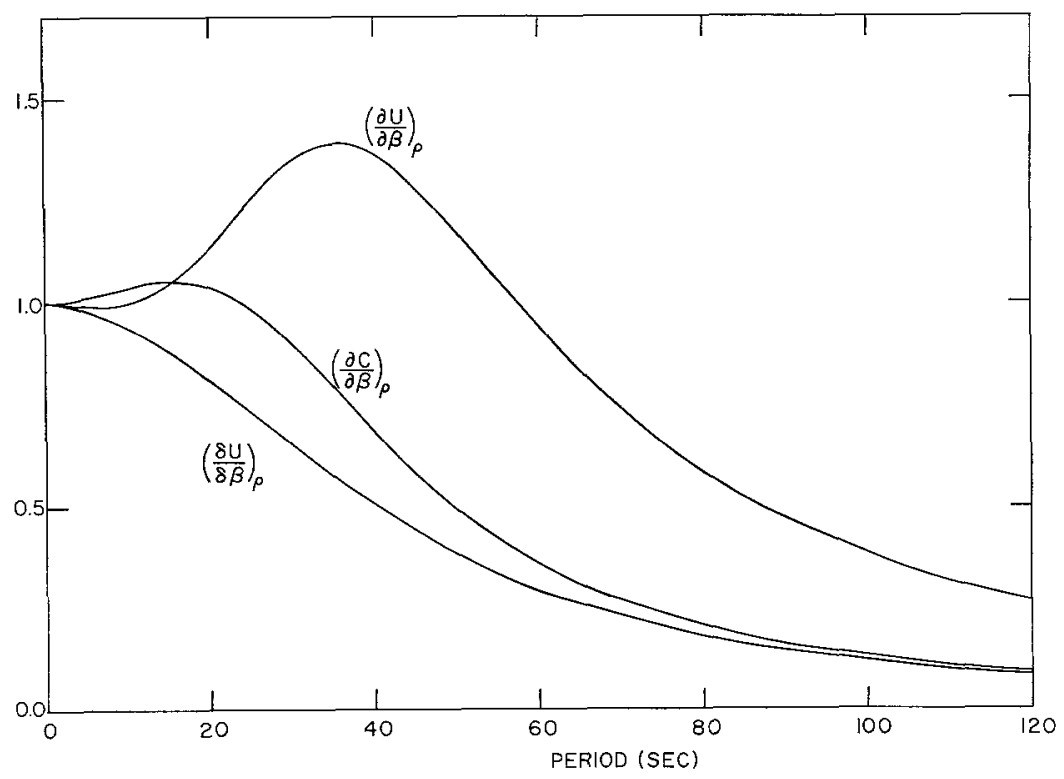

Fig. 1, First derivatives of the phase, $\mathrm{C}$, and group, $\mathrm{U}$, velocities of the fundamental Love wave mode with respect to the surface layer shear velocity. 


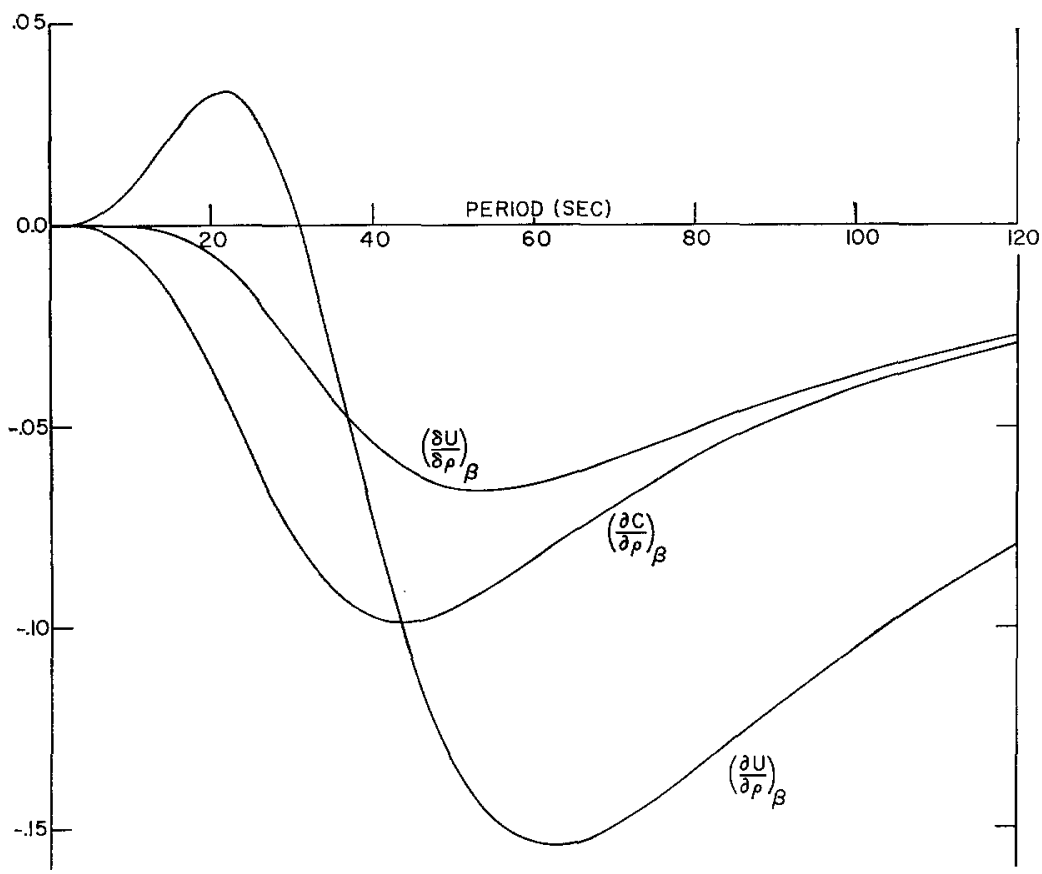

Fig. 2. First derivatives of the phase, $\mathrm{C}$, and group, $\mathrm{U}$, velocities of the fundamental Love wave mode with respect to surface layer density.

technique. The period equation (45) for the layer over a half-space can be rewritten as

$$
k=\frac{n \pi+D}{r_{\beta \mathbf{1}} d_{1}}
$$

where

$$
D=\arctan B
$$

and

$$
B=\frac{\mu_{2}}{\mu_{1}}\left[\frac{1-\left(\frac{c}{\beta_{2}}\right)^{2}}{\left(\frac{c}{\beta_{1}}\right)^{2}-1}\right]^{1 / 2} .
$$

Restricting our formulas to the fundamental mode, i.e., $n=0$, we have

$$
k=\frac{\mathfrak{D}}{r_{\beta 1} \bar{d}_{1}}
$$

and

$$
U=\frac{d \omega}{d k}=c+k \frac{d c}{d k}=c+\frac{k}{G}
$$




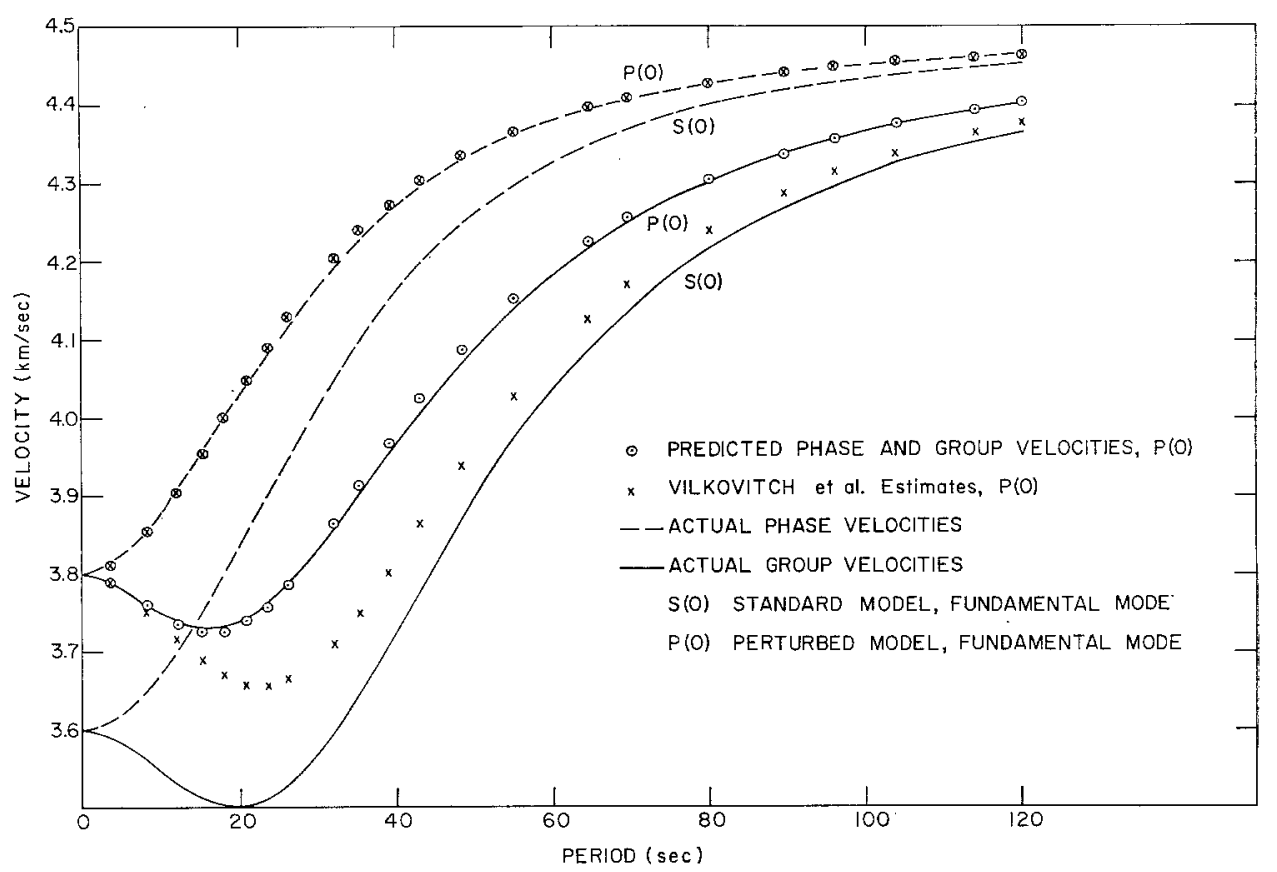

FIG. 3. Standard and perturbed model phase and group velocities with predicted values based on first derivatives.

where

$$
G \equiv \frac{d k}{d c}
$$

The explicit formula for $k$, equation (60), can be differentiated with respect to $c$ to give

$$
G=\frac{1}{r_{\beta 1} d_{1}}\left[\frac{E}{1+B^{2}}-\frac{c D}{\left(\beta_{1} r_{\beta 1}\right)^{2}}\right]
$$

where

$$
E \equiv-c B\left\{\frac{1}{\left(\beta_{2} r_{\beta 2}^{*}\right)^{2}}+\frac{1}{\left(\beta_{1} \gamma_{\beta 1}\right)^{2}}\right\}
$$

With these expressions we have $k$ and $U$ as explicit functions of $c$. We can now use the following formulas to calculate the phase-velocity perturbations

$$
\left(\frac{\partial k}{\partial a}\right)_{\omega}=\frac{k}{U} \frac{d c}{d k}\left(\frac{\partial k}{\partial a}\right)_{c}=\frac{k}{U G}\left(\frac{\partial k}{\partial a}\right)_{c}
$$

and

$$
\left(\frac{\partial c}{\partial a}\right)_{\omega}=-\frac{c}{U G}\left(\frac{\partial k}{\partial a}\right)_{c}
$$


where $a$ can be either $\rho, \mu$, or $\beta$. From the explicit relations, we obtain

$$
\left(\frac{\partial k}{\partial \mu_{1}}\right)_{c}=-\frac{1}{r_{\beta 1} d_{1}}\left\{\frac{D_{c}^{2}}{2 \mu_{1} \beta_{1}^{2} r_{\beta 1}}-\frac{1}{1+B^{2}} \frac{B}{2 \mu_{1}}\left[2-\frac{c^{2}}{\left(\beta_{1} r_{\beta 1}\right)^{2}}\right]\right\}
$$

TABLE 3

Phase Velocities (c) and Amplitude Responses (A) For the Perturbed Model with their Estimates ( $c^{*}$ and $\mathrm{A}^{*}$ ) USing Derivatives

$\begin{array}{rcccc}\mathrm{T}(\mathrm{sec}) & \mathrm{e}(\mathrm{km} / \mathrm{sec}) & \mathrm{c}^{*}(\mathrm{~km} / \mathrm{sec}) & \mathrm{A}\left(10^{-15} \mu / \mathrm{dy}\right) & \mathrm{A}^{*}\left(10^{-15} \mu / \mathrm{dy}\right) \\ 120.13 & 4.4678 & 4.4674 & 0.2115 & 0.2127 \\ 113.90 & 4.4643 & 4.4639 & 0.2353 & 0.2362 \\ 103.83 & 4.4573 & 4.4570 & 0.2830 & 0.2828 \\ 95.99 & 4.4504 & 4.4501 & 0.3309 & 0.3293 \\ 89.65 & 4.4435 & 4.4434 & 0.3791 & 0.3755 \\ 79.90 & 4.4298 & 4.4300 & 0.4759 & 0.4672 \\ 69.64 & 4.4095 & 4.4105 & 0.6226 & 0.6032 \\ 64.51 & 4.3961 & 4.3978 & 0.7214 & 0.6930 \\ 55.06 & 4.3629 & 4.3665 & 0.9718 & 0.9159 \\ 48.35 & 4.3299 & 4.3356 & 1.2275 & 1.1399 \\ 43.16 & 4.2967 & 4.3045 & 1.4889 & 1.3700 \\ 38.90 & 4.2632 & 4.2728 & 1.7566 & 1.6105 \\ 35.24 & 4.2290 & 4.2399 & 2.0311 & 1.8647 \\ 28.99 & 4.1577 & 4.1693 & 2.6018 & 2.4204 \\ 23.45 & 4.0810 & 4.0908 & 3.2041 & 3.0355 \\ 18.01 & 3.9973 & 4.0035 & 3.8422 & 3.6971 \\ 11.96 & 3.9046 & 3.9069 & 4.5395 & 4.4107 \\ 6.70 & 3.8385 & 3.8389 & 5.0962 & 4.9676 \\ 3.45 & 3.8114 & 3.8144 & 5.4205 & 5.2880\end{array}$

TABLE 4

Group Velocities (U) for the Perturbed Model with their Estimates, $\mathrm{U}^{*}$, Using Derivatives and, V, Using

Vilkovitch's Formulla

$\begin{array}{rccc}\mathrm{T}(\mathrm{sec}) & \mathrm{U}(\mathrm{km} / \mathrm{sec}) & \mathrm{U}^{*}(\mathrm{~km} / \mathrm{sec}) & \mathrm{V}(\mathrm{km} / \mathrm{sec}) \\ 120.13 & 4.4060 & 4.4056 & 4.3793 \\ 113.90 & 4.3961 & 4.3959 & 4.3663 \\ 103.83 & 4.3766 & 4.3768 & 4.3406 \\ 95.99 & 4.3575 & 4.3583 & 4.3153 \\ 89.65 & 4.3388 & 4.3403 & 4.2904 \\ 79.90 & 4.3024 & 4.3056 & 4.2419 \\ 69.64 & 4.2506 & 4.2568 & 4.1725 \\ 64.51 & 4.2177 & 4.2260 & 4.1286 \\ 55.06 & 4.1410 & 4.1542 & 4.0275 \\ 48.35 & 4.0715 & 4.0880 & 3.9392 \\ 43.16 & 4.0087 & 4.0263 & 3.8638 \\ 38.90 & 3.9521 & 3.9686 & 3.8010 \\ 35.24 & 3.9015 & 3.9151 & 3.7503 \\ 28.99 & 3.8184 & 3.8234 & 3.6833 \\ 23.45 & 3.7608 & 3.7583 & 3.6578 \\ 18.01 & 3.7326 & 3.7272 & 3.6696 \\ 11.96 & 3.7404 & 3.7369 & 3.7161 \\ 6.70 & 3.7707 & 3.7698 & 3.7654 \\ 3.45 & 3.7900 & 3.7899 & 3.7892\end{array}$




$$
\left(\frac{\partial k}{\partial \rho_{1}}\right)_{c}=\frac{1}{r_{\beta 1} d_{1}}\left[\frac{D c^{2}}{2 \mu_{1} r_{\beta 1}}-\frac{1}{1+B^{2}} \frac{B}{2 \rho_{1}} \frac{c^{2}}{\left(\beta_{1} r_{\beta 1}\right)^{2}}\right] .
$$

For calculating the perturbations of $U$ we used

$$
\left(\frac{\partial U}{\partial a}\right)_{\omega}=\left(\frac{\partial U}{\partial a}\right)_{c}+\left(\frac{\partial U}{2 c}\right)_{a}\left(\frac{\partial c}{\partial a}\right)_{\omega}
$$

and the explicit function of $U$ in terms of $c$ and the physical constants $a$.

The phase- and group-velocity perturbations for the standard model are shown

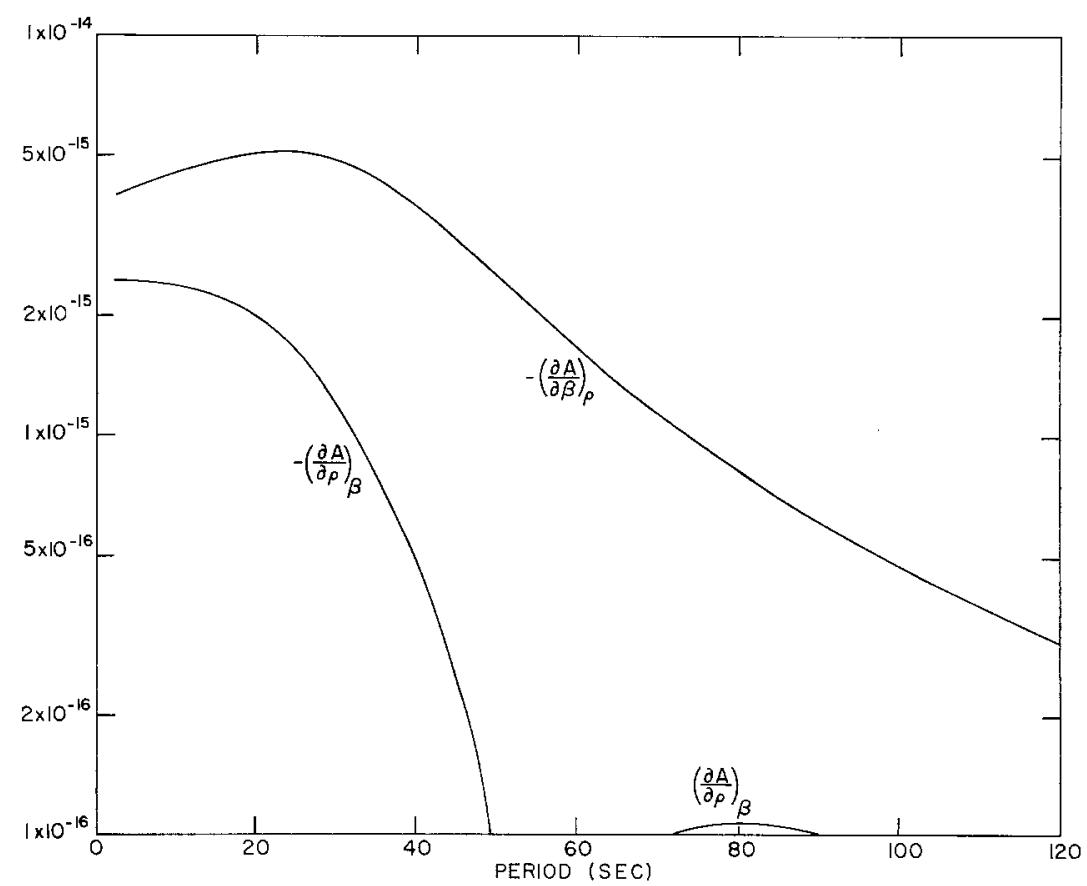

FIG. 4. First derivatives of the amplitude response, A, of the fundamental Love wave mode with respect to surface layer shear velocity and density.

in Figures 1 and 2. The actual group velocity perturbations are seen to differ considerably from the Vilkovitch et al (1966) values $\left(\delta U / \delta \beta_{1}\right)_{\rho}$ and $\left(\delta U / \delta \rho_{1}\right)_{\beta}$. These values were calculated using the formulas in Vilkovitch et al (1966) and agree with their plotted values. The group-velocity derivatives with respect to the surface-layer shear velocity are actually greater than the corresponding phase-velocity derivatives.

An interesting result not predicted from the incorrect group-velocity derivatives: is that for high frequencies the group-velocity perturbations differ in sign with the phase-velocity perturbations due to a change in surface density. Increasing the density, increases the group velocity at periods less than 30 seconds while decreasing it at greater periods. 


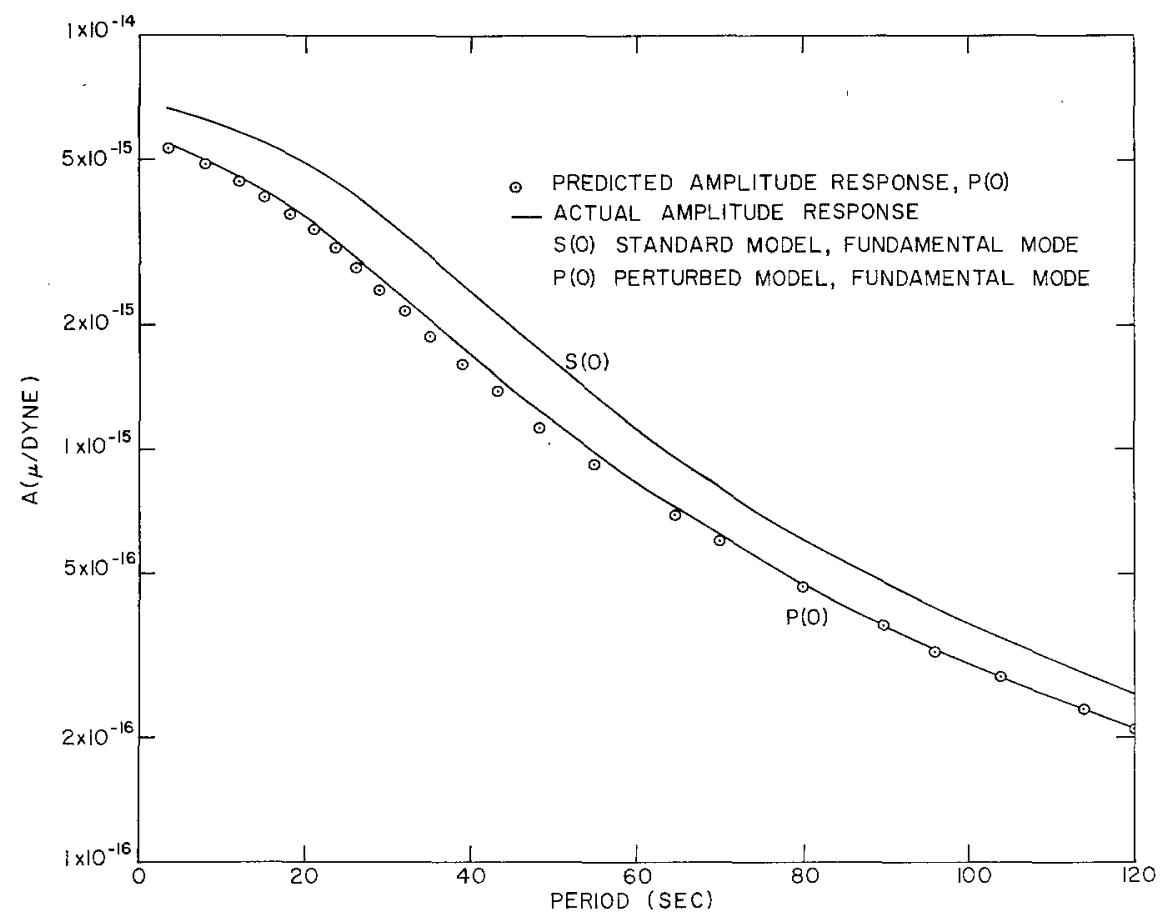

Frg. 5. Standard and perturbed model amplitude responses with predicted valves based on first derivatives.

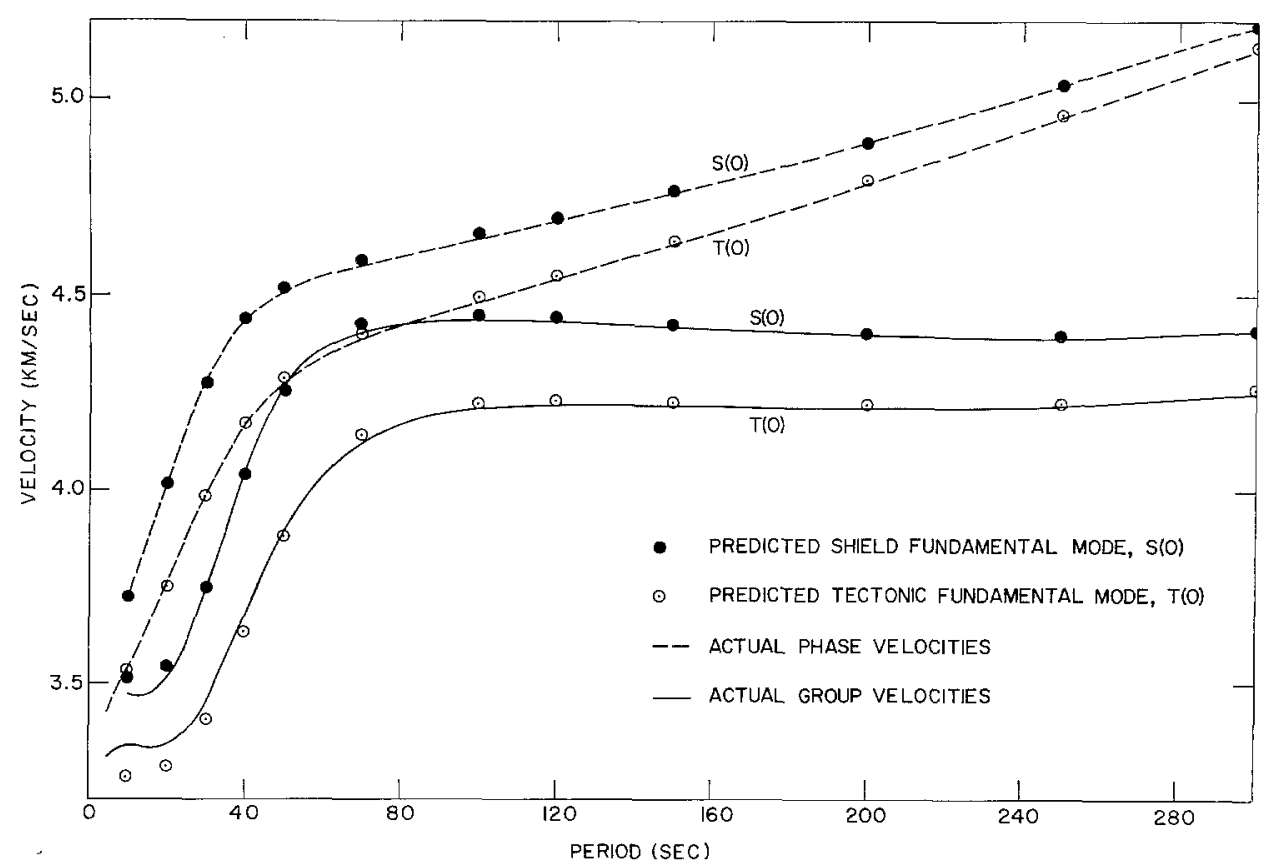

FIG. 6. Shield and Tectonic model phase and group velocities with predicted values based on first derivatives. 
In Figure 3 the phase and group velocity are shown for the standard model. In order to demonstrate the effectiveness of using the partial derivatives to estimate the dispersion of similar models, we have calculated the exact and estimated spectra for the perturbed model in Table 1. The estimated and exact values of the phase and group velocity for this model are given in Tables 3 and 4 and are shown in Figure 3. Group velocity estimates based on the Vilkovitch values are given in Table 4 and Figure 3.

From Figure 3, we see that estimates based on first derivatives are very good. Values from Vilkovitch (1966) give poor estimates of the new group velocity. As an example of the technique they calculated the phase and group velocities for a perturbation of the standard model to a model with constant velocity and density

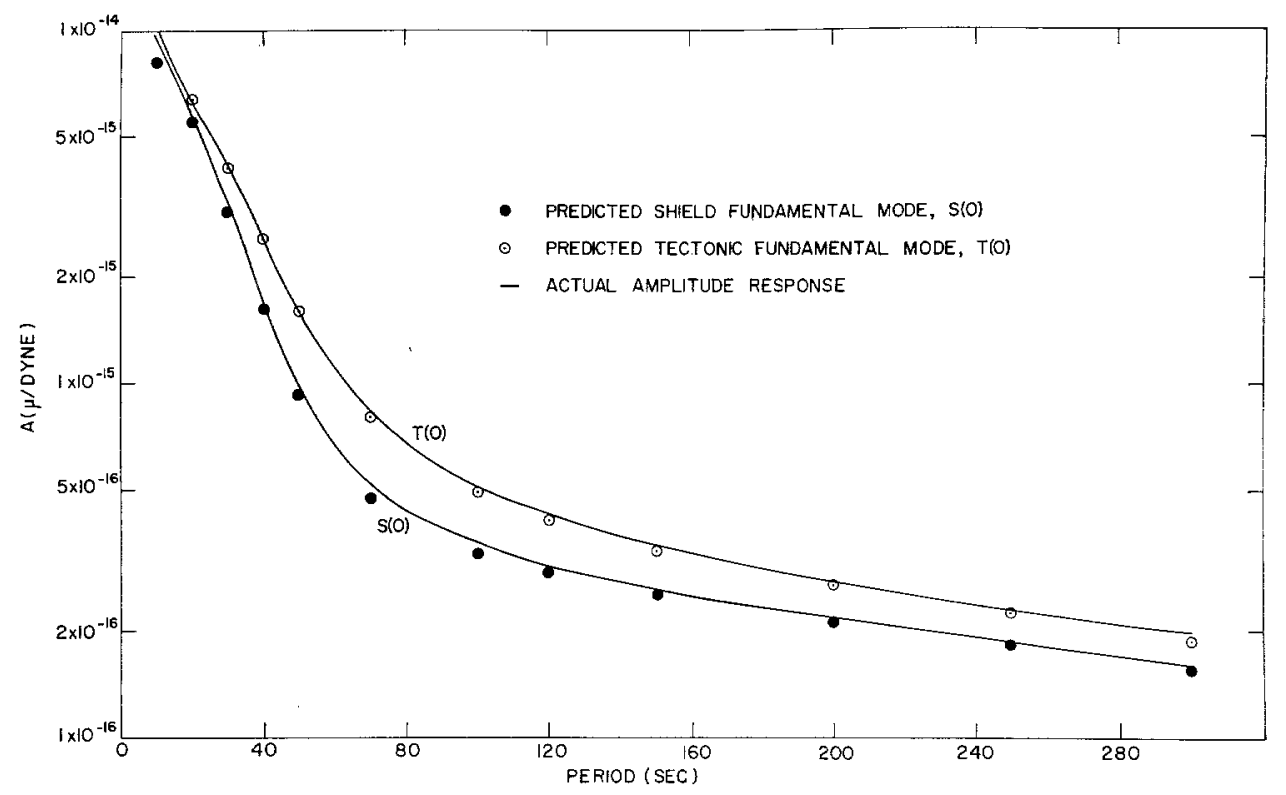

Frg. 7. Shield and Tectonic model amplitude responses with predicted values based on first derivatives.

gradients in the layer and half-space. Even though they made a correction to the values for constant velocity and density perturbations, it is surprising that their group velocity estimates were as good as shown in their figure.

In Figures 4 and 5, the amplitude response perturbations are given along with the resulting estimates for the perturbed model.

For the multilayered half-space and plate, the derivatives were calculated approximately by a difference scheme and analytically by using a tedious but straightforward method described in Anderson and Harkrider (1968). The technique is essentially the $n$ layer extension of equations (44) through (48). Again the assumptions of Anderson (1964), Andrianova et al (1965) and Vilkovitch et al (1966) were found to be inapplicable.

Two multilayered models along with their mutual spectral estimates are given in Figures 6 and 7 . The models and the partial derivative tables used to calculate their 
predicted spectra can be found in Anderson and Harkrider (1968). The agreement between predicted and actual spectral values for the fundamental modes shown in these figures is extremely good.

\section{ACKNOWLEDGMENTS}

This research was supported by the Advanced Research Projects Agency and was monitored by the Air Force Office of Scientific Research under contract AF49 (638)-1693.

\section{REFERENCES}

Anderson, Don L. (1964). Universal dispersion tables, I, Love waves across oceans and continents on a spherical Earth, Bull. Seism. Soc. Am. 54, 681-726.

Anderson, Don L. and David G. Harkrider (1968). Universal dispersion tables, II. Variational parameters for amplitudes, phase velocity and group velocity for first four Love modes for an oceanic and continental Earth model, submitted to Bull. Seism. Soc. Am.

Andrianova, Z. S., V. I. Keilis-Borok, A. L. Levshin, and M. G. Neigauz (1965). Seismic Love Waves, Nauka Press, Moscow. English translation by Consultants Bureau, Plenum Publishing Corporation, New York.

Backus, G. E. and J. F. Gilbert (1967). Numerical Applications of a Formalism for Geophysical Inverse Problems, Geophys. $J ., 13,247-276$.

Harkrider, David G. (1964). Surface waves in multilayered elastic media, 1. Rayleigh and Love waves from buried sources in a multilayered elastic half-space, Bull. Seism. Soc. Am. 54, 627-679.

Harkrider, David G. and Don L. Anderson (1966). Surface wave energy from point sources in plane layered Earth models, J. Geophys. Res. 71, 2967-2980.

Jeffreys, H. (1934). The surface waves of earthquakes, Monthly Notices, Roy. Astron. Soc., Geophys. Suppl. 3, 253-261.

Jeffreys, H. (1961). Small corrections in the theory of surface waves, Geophys. J. 6, 115-117.

Keilis-Borok, V. I. and T. B. Yanovskaya (1962). Dependence of the spectrum of surface waves on the depth of the focus within the Earth's erust, Bull. Acad. Sci., USSR, Geophys. Ser., English Transl. 11, 1532-1539.

Kovach, R. L. and Don L. Anderson (1964). Higher mode surface waves and their bearing on the structure of the Earth's mantle, Bull. Seism. Soc. Am. 54, 161-182.

Meissner, E. (1926). Elastiche oberflächen Querwellen, Verhandl. Intern. Kongr. Tech. Mech., 2nd, Zurich, 3-11.

Saito, M. (1967). Excitation of free oscillations and surface waves by a point source in a vertically heterogeneous Earth, $J$. Geophys. Res. 72, (in press).

Takeuchi, H., M. Saito and N. Kobayashi (1962). Study of shear velocity distribution in the upper mantle by mantle Rayleigh and Love waves, J. Geophys. Res. 67, 2831-2839.

Takeuchi, H., J. Dorman and M. Saito (1964). Partial derivatives of surface wave phase velocity with respect to physical parameter changes within the Earth, J. Geophys. Res. 69. 3429-3441.

Vilkovitch, E. V., A. L. Levshin and M. G. Neigaus (1966). Love Waves in a Vertically Inhomogeneous Medium, Machine Interpretation of Seismic Waves, Electronic Computer Seismology, Part 2, 130-149.

Vlaar, N. J. (1966). The field from an $S H$ point source in a continuously layered inhomogeneous half-space. Part II. The field in a half-space, Bull. Seism. Soc. Am. 56, 1305-1316.

Department of Geological Sciences

BROWN UNIVERSITY

Providence, Rhode Istand

Manuscript received August 31, 1967. 